\title{
Some Experimental Results Concerning Nonreciprocal East-West VLF Wave Propagation
}

\author{
B. Burgess
}

Ministry of Aviation, Royal Aircraft Establishment, Radio Department, S. Farnborough, Hampshire, England

\section{Introduction}

Experimental data collected in recent years by Crombie [1958], and Reder et al. [1962], and theoretical work on VLF propagation by Wait [1960] have given strong evidence to the fact that VLF propagation is nonreciprocal in the east-west and west-east directions. Experimental data relating to this topic are at present sparse. This paper presents some experimental results obtained during the past 2 years which will substantiate the existing evidence as to this nonreciprocal propagation.

Since December 1961, the phase of the VLF transmitter NPM $(19.8 \mathrm{kc} / \mathrm{s})$ located at Hawaii has been monitored and compared with a local high stability frequency source in Singapore, which is approximately $11,000 \mathrm{~km}$ away. The shortest propagation path is one which travels within $20 \mathrm{deg}$ of an east to west direction. The relative phase of GBR (distance 11,000 km) has also been monitored over the same period.

While the diurnal variation of phase of GBR $(16.0 \mathrm{kc} / \mathrm{s})$ at this site has undergone the usual trapezoidal pattern with a day to night excursion of 1.1 to 1.3 cycles of phase, the diurnal phase of NPM has exhibited a double-humped pattern regularly day after day (fig. 1). It is proposed in this paper that this form of diurnal phase variation is due to the nonreciprocity of VLF propagation in the east-west and west-east directions. Further evidence to support this conclusion has been obtained from:

(a) short term monitoring of NPM at Gan in the Maldive Islands, and

(b) from flight measurements of several thousand

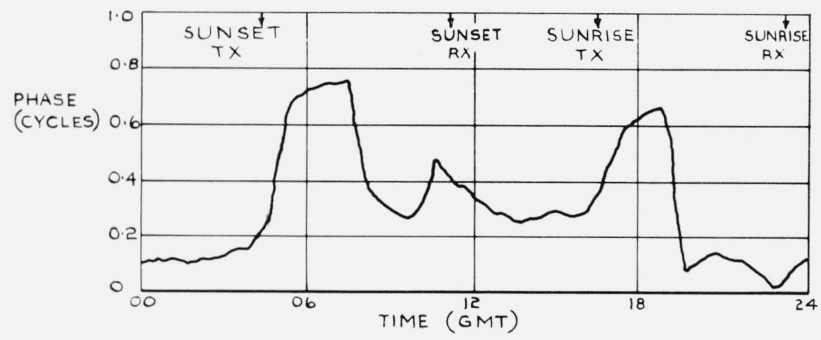

FIGURE 1. Diurnal variation of phase of NPM $(19.8 \mathrm{kc} / \mathrm{s})$ recorded at Singapore. kilometers from Singapore in the direction of Hawaii.

The limit in geographic extent of this type of phenomena is important for long range navigation systems using VLF waves.

\section{Evidence of Nonreciprocity}

The first evidence of nonreciprocity of VLF propagation in the east-west direction was first put forward by Round at al. [1925]. Crombie [1958] drew attention back to this work, and with his own measurements on the field strength of GBR (Rugby), monitored in New Zealand, put forward more conclusive evidence that the effect did exist. Reder et al. [1962] have shown from flight measurements that there is a very small east-west nonreciprocity in the phase velocity of VLF waves, but phase on theoretical grounds [Wait, 1960] is expected to be much less affected than signal strength by nonreciprocal propagation.

Barber and Crombie [1959] and Wait [1960] have shown theoretically that the attenuation in the east-west direction is markedly greater than in the west-east direction. From the published data of Wait [1960], it is quite possible to choose a waveguide model such that the attenuation for an eastwest path is over three times that of a corresponding: west-east path.

Taking the NPM/Singapore path as an example, where the short path is $11,000 \mathrm{~km}$ and the long path $29,000 \mathrm{~km}$, it is thus possible for the long path to have the larger signal. The time of day will, of course, cause the signal strength to vary and the possibility of both paths being dominant at different times of day is highly likely.

\section{Results of Measurements}

The diurnal variation of phase for NPM at Singapore is shown in figure 1. Also marked on the diagram are the times of sunset and sunrise at the transmitter (NPM) and the receiver (Singapore). This type of variation is typical of all seasons, the only variation being that the peaks in the pattern may be diminished during certain periods of the year. It is evident that the phase pattern cannot be explained by day to night variations in the 11,000 $\mathrm{km}$ propagation path alone between Hawaii and 


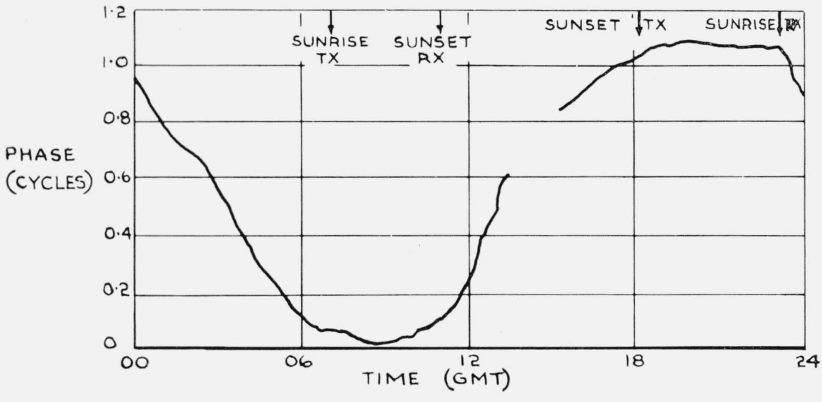

Figure 2. Diurnal variation of phase of $G B R\left(\begin{array}{lll}16 & k c / s\end{array}\right)$ recorded at Singapore.

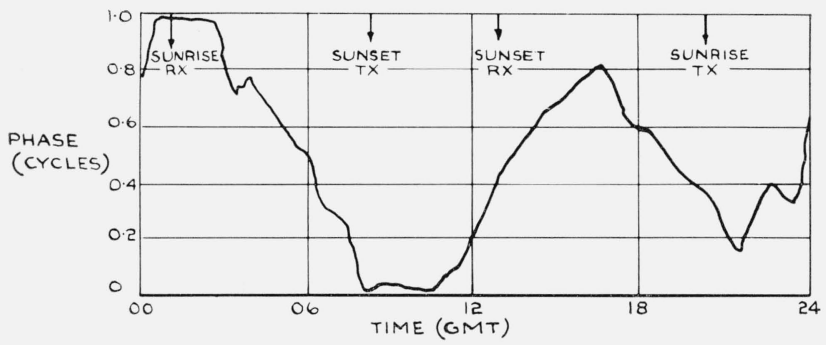

Figure 3. Diurnal variation of phase of NPM $(19.8 \mathrm{kc} / \mathrm{s})$ recorded at Gan, Maldive Islands.

Singapore. If that single propagation path were present the whole $24 \mathrm{hr}$, one would expect a phase pattern similar to that of GBR recorded at singapore (fig. 2).

The next simple model to put forward is that during one 12 -hr period, the long path is dominant, and during the next 12-hr period, the short path dominates. The resultant phase plot during the day would thus consist of a combination of two trapezoidal patterns, $12 \mathrm{hr}$ out of phase with one another, with the appropriate parts of the two phase patterns forming the resultant. From this model the appearance of two peaks in the recorded diurnal phase plot can be explained, though the actual times of occurrence of these peaks do not quite agree with the expected times from this simple model.

In order to test the extent of this type of phenomena, NPM has on two separate occasions, of a few days each, been monitored at Gan in the Maldive Islands. Gan is situated in the Indian Ocean, some 14,000 km from Hawaii, and lying more or less on the great circle path through Hawaii-Singapore. Figure 3 shows the phase plot of NPM as obtained at Gan. The same general feature as found at Singapore are present, giving added confidence to the Singapore measurements.

Flight measurements of the phase of MPM between Singapore, Manila, and Hong Kong have been made recently and an initial analysis indicates that the signal during certain parts of the flight was being received over the long propagation path $(30,000 \mathrm{~km})$ showing the preference of west-east propagation.

\section{Concluding Remarks}

There is strong evidence that the results presented above are due to nonreciprocity in the eastwest, west-east propagation of VLF waves. The range of propagation phenomena exist at least to within $10,000 \mathrm{~km}$ of the transmitter. Plans are being made to monitor at closer ranges in order to determine when the short path is dominant all the day. Further, monitoring of NPM at Nairobi $(17,000 \mathrm{~km}$ from Hawaii) will give an indication of the dominance of the long path throughout the day. From these measurements it should be possible to estimate difference in attenuation between east-west and west-east paths.

\section{References}

Barber, N. F., and D. D. Crombie (1959), VLF reflections from the ionosphere in the presence of a transverse magnetic field, J. Atmos. Terres. Phys. 16, 37.

Crombie, D. D. (1958), Differences between the east-west and west-east propagation of VLF signals over long distances, J. Atmos. Terres. Phys. 12, 110.

Reder, F. H., and G. M. R. Winkler (1963), Nonreciprocity, dispersion, diurnal behaviour and precision of frequency transfer of VLF propagation, presented at VLF Symposium, 12, 13, and 14 August, Boulder, Colo.

Round, H. J., T. L. Eckersley, K. Tremellen, and F. C. Lunnon (1925), Report on measurements made on signal strength at great distances during 1922 and 1923 by an expedition sent to Australia.

Wait, J. R., and Kenneth Spies (1960), Influence of earth curvature and the terrestrial magnetic field on VLF propagation, J. Geophys. Res. 65, 2325.

(Paper 68D1-310) 